\title{
In Silico Assessment of Nifedipine Effects on Human Heart Cells: Pharmacokinetic-Pharmacodynamic Analyses at the Population Level
}

\author{
Mitra Abbasi ${ }^{1}$, Sebastian Polak ${ }^{1,2}$ \\ ${ }^{1}$ Simcyp Limited (a Certara Company), Blades Enterprise Centre, Sheffield, UK \\ ${ }^{2}$ Faculty of Pharmacy, Jagiellonian University Medical College, Krakow, Poland
}

\begin{abstract}
This study aimed to utilise the value of integrating in vitro data and physiologically based pharmacokinetic $(P B P K)$ models to quantitatively estimate the impact on pharmacokinetics $(P K)$ and pharmacodynamics $(P D)$. The objective was to predict pharmacodynamics (electrocardiogram (ECG) parameters) of nifedipine (NIF) after an oral administration by simulation. The computational models were performed for human transmural ECGs to model drug-induced changes in $Q T$ interval as well as changes in T-wave morphology.

The differences in QTc interval due to NIF in healthy volunteers both males and females were predicted by the Cardiac Safety Simulator, providing a mechanistic understanding of clinical observation. The simulation results showed that NIF significantly shortened the QTCF in a concentration-dependent manner compared with baseline (placebo) control. Comparison of the simulated QT interval of non-QT-prolonging drug (NIF) against the clinical observations proved the accuracy of the model based prediction.
\end{abstract}

\section{Introduction}

This study was designed to evaluate the PD effect of NIF on human cardiac myocytes. NIF is a $\mathrm{Ca}^{2+}$ channel blocker used in the treatment of various cardiovascular diseases, with the pharmacological target in the vascular smooth muscles [1]. In the current study, the PK-PD models were developed to investigate the PD changes in relation to the plasma drug concentrations. Two case studies of the PK-PD relation of NIF were analyzed: The first simulation study was designed using the drug exposure compound file and population data available in the Simcyp simulator, and plasma concentration data was exported from Simcyp to the Cardiac Safety Simulator (CSS) in order to analyse the PK-PD effect of the compound at the population level. In the second case a clinical study was mimicked [2] by utilizing CSS to support in silico assessment.

\section{Methods}

Drug pharmacokinetics (PK) was assessed with the use of the Simcyp population-based simulator (V15R1 www.simcyp.com; [3]). The NIF compound file and population data [4] available in Simcyp (V15) were used for all simulations. NIF is an L-type calcium channel blocker used for the treatment of hypertension and angina pectoris. To assess the proarrhythmic potency of the drug, the Cardiac Safety Simulator (CSS V2.1, Simcyp, Sheffield) was used to evaluate pharmacodynamics (the cardiac electrophysiological effects) of NIF in a virtual human population of European Caucasians (NEurCaucasian). The substrate and trial design input parameters are presented in Table 1 and 2 respectively. Inhibitory activity (i.e. the dose-inhibition relationship) of this compound (Table 3) were specified for ion channels by using multiple free (unbound) drug concentrations expressed in micromoles $(\mu \mathrm{M})$. The computations were performed for human transmural ECGs to model druginduced changes in QT interval as well as changes in Twave morphology (PD endpoints). The modelling approach for this study is described in Figure 1. This diagram illustrates the PK-PD relationships (using Simcyp and CSS simulators) in which pharmacodynamics examine the time course of the biological effects of the drug. The mechanism of drug action related to concentration in plasma can be predicted using a PBPK modeling approach.

\subsection{Model Input Data}

NIF compound (Table 1) was selected for investigation for its pure $\mathrm{Ca}^{2+}$ channel blocking properties. The drug plasma concentration is taken from the PBPK model and utilised as an input to the CSS. The half-maximal inhibitory concentration (IC50) and Hill coefficient (h) data (Table 3) describing drug triggered ionic current modification were used as input parameters in addition to multiple free (unbound) drug plasma concentrations $(\mu \mathrm{M})$.

Table 1. Input parameters (substrate) used in Simcyp population-based simulator (V15 R1)

\begin{tabular}{|l|l|}
\hline & Substrate \\
\hline Compound Name & Sim-Nifedipine \\
\hline $\begin{array}{l}\text { Molecular Weight } \\
(\mathrm{g} / \mathrm{mol})\end{array}$ & 346.3 \\
\hline
\end{tabular}




\begin{tabular}{|l|l|}
\hline $\log \mathrm{P}$ & 2.690 \\
\hline Compound Type & Monoprotic Base \\
\hline $\mathrm{pKa}$ & 2.820 \\
\hline fu & 0.039 \\
\hline Absorption Model & 1 st order \\
\hline Distribution Model & Minimal PBPK Model \\
\hline Vss (L/kg) & 0.570 \\
\hline Clearance Type & Enzyme Kinetics \\
\hline Fasted/Fed & Fed \\
\hline Route & Oral \\
\hline Dose Units & Dose (mg) \\
\hline Dose & 10.0 \\
\hline Start Day/Time & Day 1, 09:00 \\
\hline Dosing Regimen & Single Dose \\
\hline
\end{tabular}

(i.e. Sim-NEurCaucasian, North European Caucasian) [8].

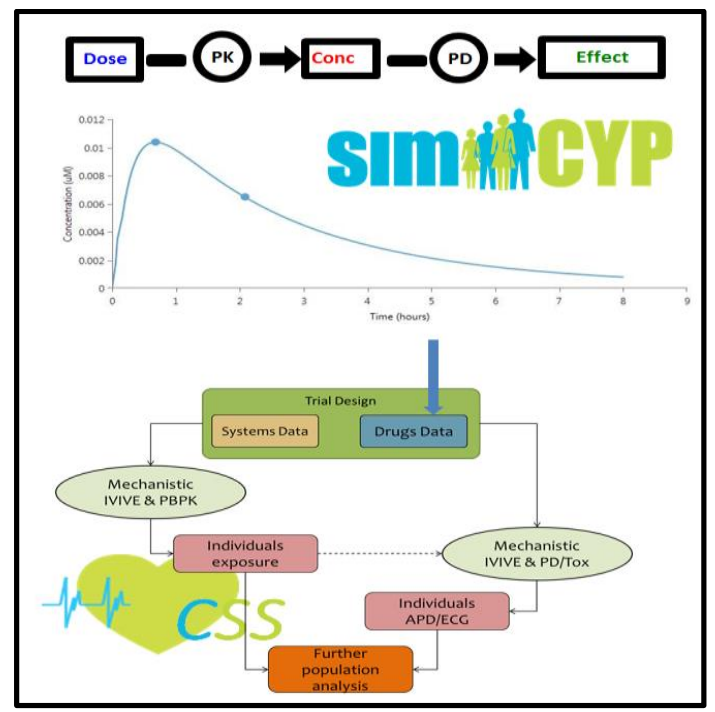

Table 2. Input parameters (trial design) used in Simcyp population-based simulator (V15 R1)

\begin{tabular}{|l|l|}
\hline Trial Design & \\
\hline Population Name & Sim-Healthy Volunteers \\
\hline Population Size & 15.00 \\
\hline Number of Trials & 3.00 \\
\hline No. of Subjects per Trial & 5.00 \\
\hline Start Day/Time & Day 1, 09:00 \\
\hline End Day/Time & Day 1, 17:00 \\
\hline Study Duration (h) & 8.00 \\
\hline Sampling Time & Pre-defined Uniform \\
\hline
\end{tabular}

Table 3. Inhibitory actions of NIF on ion channels in vitro

\begin{tabular}{|c|c|c|}
\hline \multicolumn{2}{|c|}{ Effects of NIF on cardiac transmembrane ion currents } \\
\hline \multirow{2}{*}{ Ion Channel current } & $\begin{array}{c}\text { Inhibitory Potency } \\
I_{C 50}(\mu \mathrm{M})\end{array}$ \\
\hline \multirow{2}{*}{ Inward } & $I_{N a}$ & $88.5(\mathrm{~h}=0.71)[5]$ \\
\cline { 2 - 3 } & $I_{C a L}$ & $0.012(\mathrm{~h}=1.02)[5]$ \\
\hline \multirow{2}{*}{ Outward } & $I_{K r}$ & $22^{*}(\mathrm{~h}=0.8)[5]$ \\
\cline { 2 - 3 } & $I_{K s}$ & $360(\mathrm{~h}=0.97)[6]$ \\
\hline
\end{tabular}

* Measured at room temperature and scaled down twofold.

\subsection{Human ventricular Cell model and Cardiac Safety Simulator (CSS)}

The computational models were derived from the ten Tusscher Panfilov 2006 (tT2006) human ventricular model [7] by proportionally changing ionic currents based on the in vitro measurements. To assess the antiarrhythmic potency of the NIF, the CSS platform (V2.1) was used to simulate normal (control) signal and drug-induced alterations in cardiac AP conduction at the population level

Figure 1. Population-specific simulation schematic representation of combination of PK and PD model (using Simcyp and CSS simulators).

\subsection{Simulation analysis}

The minimal PBPK Model was applied for the PK simulations in the Simcyp simulator. The PK model input parameters are presented in Tables 1 and 2. The PD effect (the electrophysiological characteristics) of NIF was conducted in the CSS simulator. The PD end points generated in CSS using tT2006 model for this study are: AP and ECG morphology, Heart Rate (HR), QTcF (QT interval corrected by heart rate using Fridericia correction method), Tpeak-Tend, JTpeak, $\Delta \mathrm{QTcF}$ (difference between the average of baseline of all placebo QTcF and $\mathrm{QTcF}$ in the presence of a drug), $\Delta$ Tpeak-Tend and $\Delta$ JTpeak.

\section{Results}

\subsection{Pharmacokinetic analysis}

The use of mechanistic physiologically based pharmacokinetic (PBPK) models allowed the in vivo extrapolation of the in vitro parameters and prediction of PK parameters. As shown in Figure 2, the systemic concentration-time profile of NIF simulated by the minimal-PBPK model is used to drive the PD response, which is the changes in cardiac electrophysiology behaviors such as the QT interval alterations. A summary of simulated PK parameters of NIF is presented in Figure 2 . The PK profile showed that the maximum concentration was $0.011 \mu \mathrm{M}$ and occurred at $0.64 \mathrm{~h}$ after the dose was given. 


\subsection{Electrophysiological effects (case study one)}

In the first case study, the PBPK model was used to simulate the PK property of NIF to predict the PD response on cardiac electrophysiological characteristics. The effect of NIF on the AP of human ventricular epicardial cells and ECG waves are shown in Figure 3. NIF shortened the APD and QTc intervals in a concentration-dependent manner. A very small increase in HR is seen in female population in Figure 4. The mechanism of the HR increment with $\mathrm{Ca}^{2+}$ channel blockers is not clearly understood, but it could be a result of the shortening of the AP and the increase in diastolic interval that allows enough time for cells to send spontaneous APs more frequently [9]. The obtained QTcF simulations results and $\Delta \mathrm{QTc}, \Delta$ Tpeak-Tend and $\Delta \mathrm{JTpeak}$ analysis are presented for both males and females separately. The negative slopes for the linear regression of $\Delta \mathrm{QTcF} / \Delta$ Tpeak-Tend/ $\Delta \mathrm{JTpeakc}$ against NIF plasma concentrations indicate that the NIF drug shortened QTc intervals. A summary of the endpoints (electrophysiological parameters) generated on Cardiac Safety Simulator at the population level are summarized in Tables 4 and 5.

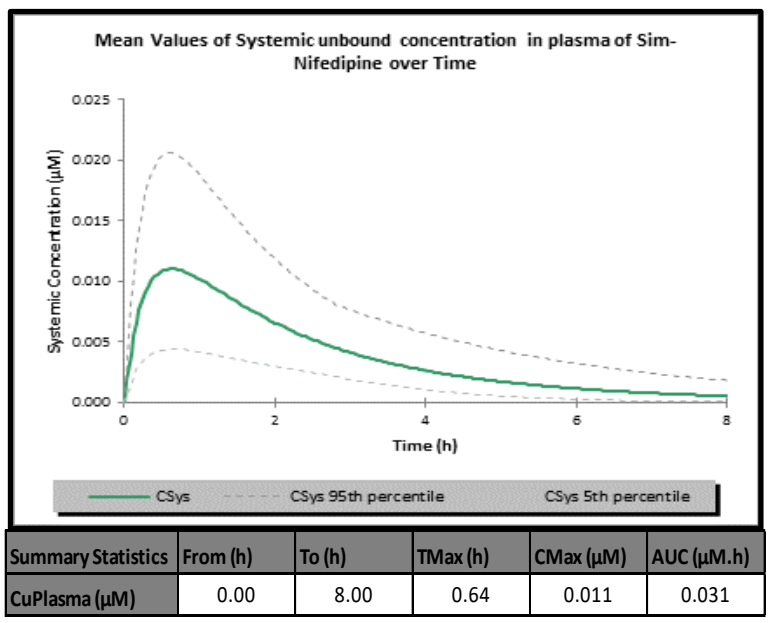

Figure 2. Simulated mean plasma concentration $(\mu \mathrm{M})$ time profile for $10 \mathrm{mg}$ single dose of NIF in healthy volunteers.

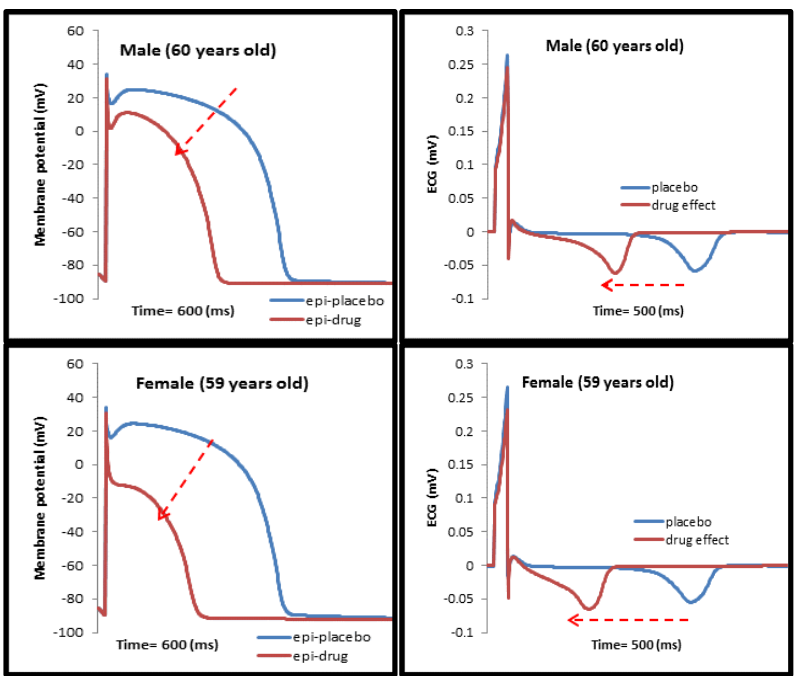

Figure 3. Concentration-dependent effects of NIF on cellular AP (left-hand side panels) and 1D ECG (righthand side) waveform conducted by using the tT2006 human (male/female) ventricular cell model. Arrows pointing into AP and ECG are showing shortening of the repolarization phase.

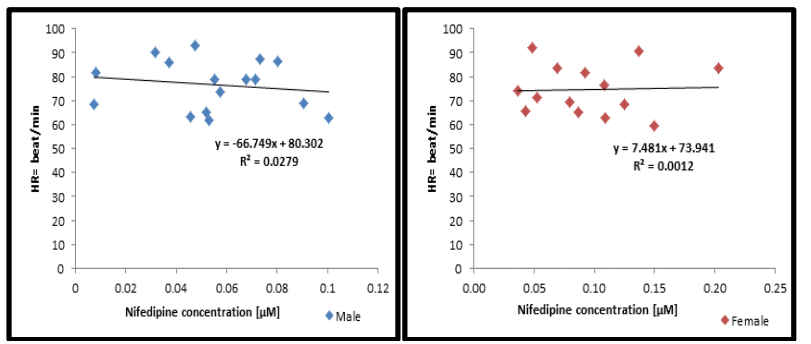

Figure 4. Concentration-dependent effects of NIF on HR (blue male, red female) at population level.

Table 4. Endpoints (ECG parameters) generated by the CSS for the placebo (base) and the presence of the drug (NIF)

\begin{tabular}{|c|c|c|c|}
\hline \multicolumn{4}{|c|}{ ECG parameters (average value (ms) of all subjects) } \\
\hline & $\begin{array}{c}\text { QTcF } \\
(\mathrm{M} / \mathrm{F})\end{array}$ & $\begin{array}{c}\text { TpeakTend } \\
(\mathrm{M} / \mathrm{F})\end{array}$ & $\begin{array}{c}\text { JTpeakc } \\
(\mathrm{M} / \mathrm{F})\end{array}$ \\
\hline base & $423.85 / 418.51$ & $59.22 / 59.3$ & $273.8 / 269.70$ \\
\hline drug & $275.1 / 242.83$ & $46.34 / 46.3$ & $162.95 / 135.31$ \\
\hline
\end{tabular}

Table 5. The percentage of the variation on the endpoints after simulation of different NIF plasma concentrations at the population level.

\begin{tabular}{|c|c|c|c|c|}
\hline \multirow{2}{*}{ sex } & \multicolumn{3}{|c|}{$\begin{array}{c}\text { *The coefficient of determination of the } \\
\text { model } R^{2} \text { (\% of the changes } \\
\text { (reduction)) }\end{array}$} & \multirow{2}{*}{$\begin{array}{c}\text { HR } \\
(\mathrm{b} / \mathrm{m})\end{array}$} \\
\cline { 2 - 4 } & $\Delta \mathrm{QTcF}$ & $\Delta$ TpeakTend & $\Delta \mathrm{JTpeakc}$ & \\
\hline $\mathrm{M}$ & $83 \%$ & $42 \%$ & $84 \%$ & 76.64 \\
\hline $\mathrm{F}$ & $50 \%$ & $61 \%$ (increased) & $69 \%$ & 74.66 \\
\hline
\end{tabular}

*CV presented here is the coefficient of variation of the 
linear regression obtained from the $\Delta \mathrm{QTcF} / \Delta \mathrm{T}$ peakTend/ $\Delta \mathrm{JT}$ peakc-concentrations plots.

\subsection{Electrophysiological effects (case study two)}

The simulation results show that NIF significantly shortened the APDs and QTc intervals (Figure 5) in a concentration-dependent manner compared with the control. The predicted values showed consistency with the experimental observations [2] in terms of significant reduction of repolarization phase of APD and QTc interval length with increasing NIF concentrations that showed the degree of the drug's influence on cardiac repolarization in healthy volunteers as compared with the control.

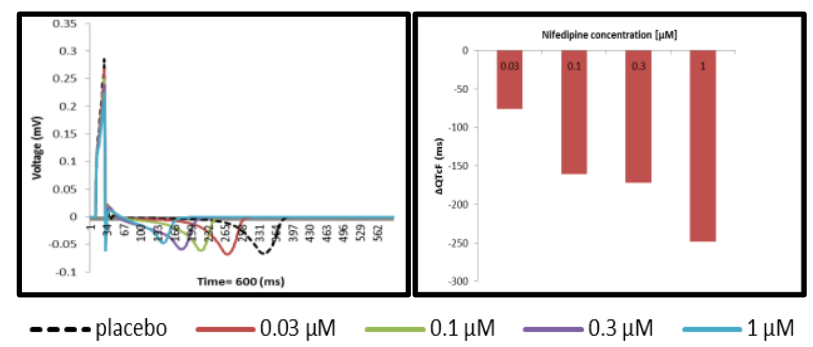

Figure 5. Left-hand side panel: the representative ECG traces of the placebo and with increasing concentrations of NIF (data taken from [2]); right-hand side panel: bar graph showing changes in $\triangle \mathrm{QTcF}$ at increasing concentrations of NIF.

\section{Discussion and conclusions}

We used PK-PD modeling and simulation to describe the relationship between individual-predicated plasma concentrations and electrophysiological changes in human. The PK of the compound was used to obtain time-matched plasma concentrations as an input to the CSS to assess cardiac electrophysiological drug effect. The first case study explored the potential of the PBPK modeling to predict the response to the cardiac electrophysiology parameters using in vitro data. The study showed the utility of PBPK-PD modeling in early cardiac safety screening in linking drug concentration at the probable site of action with toxicological and/or therapeutic effects.

The second case study was performed to mimic an experimental study (data from human-induced pluripotent stem cell-derived cardiomyocytes) [2] to represent an assay for predictive electrophysiological safety screening using in silico models. The simulation results showed that the in silico model was successful in recovering the experimental observation [2]. The simulated $\Delta \mathrm{QTcF}$ values proved that a high concentration of NIF $(1 \mu \mathrm{M})$ could inhibit at least $75 \%$ of L-type $\mathrm{Ca}^{2+}$ current [10]. In our future work, we will investigate the interaction effect of a high concentration of NIF $(1 \mu \mathrm{M})$ that might reduce proarrhythmic dose-dependent effects of drugs which increase the risk of QT interval prolongation.

\section{Acknowledgements}

This study was supported by Simcyp (a Certara Company).

\section{References}

[1] Fleckenstein A. Specific Pharmacology of Calcium in Myocardium, Cardiac Pacemakers, and Vascular Smooth Muscle. Annu Rev Pharmacol Toxicol [Internet]. 1977 Apr 1;17(1):149-66.

[2] Harris K, Aylott M, Cui Y, Louttit JB, McMahon NC, Sridhar A. Comparison of electrophysiological data from human-induced pluripotent stem cell-derived cardiomyocytes to functional preclinical safety assays. Toxicol Sci. 2013;134(2):412-26.

[3] Jamei M, Marciniak S, Feng K, Barnett A, Tucker G, Rostami-Hodjegan A. The Simcyp population-based ADME simulator. Expert Opin Drug Metab Toxicol. 2009 Feb;5(2):211-23.

[4] Jamei M, Bajot F, Neuhoff S, Barter Z, Yang J, RostamiHodjegan A, et al. A mechanistic framework for in vitro-in vivo extrapolation of liver membrane transporters: Prediction of drug-drug interaction between rosuvastatin and cyclosporine. Clin Pharmacokinet. 2014;53(1):73-87.

[5] Kramer J, Obejero-Paz CA, Myatt G, Kuryshev YA, Bruening-Wright A, Verducci JS, et al. MICE models: superior to the HERG model in predicting Torsade de Pointes. Sci Rep. 2013;3:2100.

[6] Zhabyeyev P, Missan S, Jones SE, McDonald TF. Lowaffinity block of cardiac $\mathrm{K}+$ currents by nifedipine. Eur J Pharmacol. 2000;401(2):137-43.

[7] Ten Tusscher KHWJ, Panfilov V. Alternans and spiral breakup in a human ventricular tissue model. Am J. 2006 ;291(3):H1088.

[8] Polak S, Wiśniowska B, Fijorek K, Glinka A, Mendyk A. In vitro-in vivo extrapolation of drug-induced proarrhythmia predictions at the population level. Drug Discov Today. 2014;19:275-81.

[9] Guo L, Abrams RMC, Babiarz JE, Cohen JD, Kameoka S, Sanders MJ, et al. Estimating the risk of drug-induced proarrhythmia using human induced pluripotent stem cellderived cardiomyocytes. Toxicol Sci. 2011;123(1):281-9.

[10] Rouet, R., Worou, M. E., Puddu, P. E., Lemoine, S., Plaud, B., Salle, L., ... Hanouz, J.-L. (2012). Nifedipine blocks ondansetron electrophysiological effects in rabbit purkinje fibers and decreases early after depolarization incidence. Current Clinical Pharmacology, 7(1), 41-48.

Address for correspondence.

Mitra Abbasi

Simcyp Limited (a Certara Company)

Blades Enterprise Centre, John Street, Sheffield, S2 4SU, United Kingdom

Mitra.Abbasi@certara.com 